Rapid Reviews COVID-19

\title{
Review 2: "A handheld point-of-care system for rapid detection of SARS- CoV-2 in under 20 minutes"
}

\section{Celine Perier ${ }^{1}$}

${ }^{1}$ UC Berkeley Center for Emerging and Neglected Diseases

Published on: Aug 11, 2020

DOI: $10.21428 / 2 \mathrm{e} 3983 \mathrm{f5} .52 \mathrm{fcd} 41 \mathrm{~d}$

License: Creative Commons Attribution 4.0 International License (CC-BY 4.0). 


\section{$\underline{\text { RR:C19 Evidence Scale rating by reviewer: }}$}

- Strong. The main study claims are very well-justified by the data and analytic methods used. There is little room for doubt that the study produced has very similar results and conclusions as compared with the hypothetical ideal study. The study's main claims should be considered conclusive and actionable without reservation.

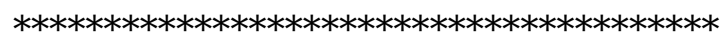

\section{Review:}

The COVID-19 pandemic has shed light of the difficulty to have a large testing capacity running. If we rely only on centralized labs with trained technicians, the issue will be even bigger. We need a molecular diagnostic solution easily accessible.

In this study Rodriguez-Manzano et al. report the development of a rapid point of care diagnostic test based on reverse transcriptase loop-mediated isothermal amplification (RT-LAMP).

The authors used the primers sequence and the LAMP assay reported by Zhang et al. in February 2020 (https://www.medrxiv.org/content/10.1101/2020.02.26.20028373v1). Their LAMP has a lower limit of detection of 10 RNA copies per reaction and was validated against 183 clinical samples including 127 positive samples (screened by the CDC RT-qPCR assay).

They were able to reach showed $90.55 \%$ sensitivity and $100 \%$ specificity when compared to RT-qPCR.

The innovative part of the study was the integration of a point-of care platform linked to a mobile device. Although their sample side was pretty limited ( $n=40$ samples), they were able to detect and quantify the presence of SARS-CoV-2 in the extracted clinical samples, with similar performance between the RT-qLAMP and the RT-eLAMP.

Although the LAMP assay by itself is not original and was already published, the authors were able:

1- To validate LAMP assay for the detection of SARS-Cov-2 with a 100\% of specificity 2 - Their point of care with integrated sensors and corresponding algorithms allow to 
detect and quantify the presence of SARS-CoV-2.

This portable diagnostic platform, is without doubt, an indispensable addition to the need of molecular diagnostic solution. 